\title{
Superconducting quantum interference devices based on YBaCuO nanobridges
}

\author{
M. V. Pedyash, ${ }^{\text {a) }}$ D. H. A. Blank, and H. Rogalla \\ Low Temperature Division, Department of Applied Physics, University of Twente, P.O. Box 217, \\ 7500 AE Enschede, the Netherlands
}

(Received 21 September 1995; accepted for publication 18 December 1995)

\begin{abstract}
Direct current SQUIDs based on $\mathrm{YBaCuO}$ thin film nanobridges have been investigated. Critical current densities $J_{c}$ of the devices are up to $3 \times 10^{6} \mathrm{~A} / \mathrm{cm}^{2}$ at $T=77 \mathrm{~K}$ and show a temperature dependence of $\left(1-T / T_{c}\right)^{1.6 \pm 0.1}$. High values of the voltage-flux modulation are observed $(8 \mu \mathrm{V}$ peak to peak at $77 \mathrm{~K}, 45 \mu \mathrm{V}$ at $4.2 \mathrm{~K}$ ). The temperature dependence of the SQUID modulation is found to be essentially different from the one of the conventional weak link SQUID. We discuss our results by considering degradation of the nanobridge area during structuring, which leads to a transition from SNS to $\mathrm{SS}^{\prime} \mathrm{S}$ type junction with decreasing temperature. (C) 1996 American Institute of Physics. [S0003-6951(96)02608-5]
\end{abstract}

Most high- $T_{c}$ SQUIDs are based on grain boundary weak links or ramp-type junctions. The techniques to prepare such structures have been developed extensively and often face technological problems caused by an extremely short coherence length $\xi$ in high- $T_{c}$ superconductors. Recent results show that nanobridges, although having dimensions significantly exceeding $\xi$, manifest a true Josephson behavior and are suitable for SQUID fabrication. ${ }^{1-3}$ The concept of the coherent motion of flux quanta has become a standard for nanobridge description, and explains a wide variety of effects. ${ }^{4}$ However, the periodic supercurrent-phase relation, which is a principal element of SQUID operation, does not follow obviously from this model.

We investigated SQUIDs based on high- $T_{c}$ thin film nanobridges systematically. We explain the Josephson nature of these devices by considering degradation of the bridge area during structuring, which leads to a transition from SNS- to SS'S-type junctions with decreasing temperature.

SQUIDs were structured in $50 \mathrm{~nm}$ thick $\mathrm{YBa}_{2} \mathrm{Cu}_{3} \mathrm{O}_{7-\delta}$ films by electron beam lithography (EBL) and direct focused ion beam milling (FIB). An inductively shunted dc SQUID geometry was chosen. ${ }^{3}$ The $T_{c}$ of unstructured films is $(90 \pm 1) \mathrm{K}$. The superconducting transition curves $R(T)$ of the investigated devices show the presence of a "foot" that grows rapidly with the decrease of the bridge width $w$, with an onset at $w \approx 250 \mathrm{~nm}$ [Fig. 1(b)]. For wider bridges no degradation of $T_{c}$ has been observed. The current voltage characteristics $(I-V)$ of SQUIDs at zero external magnetic field [see Fig. 1(a)] are similar to those of single nanobridges discussed elsewhere. ${ }^{5}$ The critical current $I_{c}$ is a linear function of the nanobridge width $w$ for $50 \mathrm{~nm}<w<350 \mathrm{~nm}$. The critical current density $J_{c}$ is up to $3 \times 10^{6} \mathrm{~A} / \mathrm{cm}^{2}$ at $77 \mathrm{~K}$ and follows $J_{c} \propto\left(1-T / T_{c}\right)^{1.6 \pm 0.1}$ in a wide range of temperatures from $T_{c}$ down to at least $T_{c} / 2$.

For $w<300 \mathrm{~nm}$, voltage-flux modulation was observed in our SQUIDs. For a device based on $250 \mathrm{~nm}$ bridges, the maximum measured peak-to-peak voltage modulation $U_{\text {mod }}$ is $8 \mu \mathrm{V}$ at $77 \mathrm{~K}$ (see Fig. 2). For a SQUID with $100 \mathrm{~nm}$ bridges $\left(T_{c}=60 \mathrm{~K}\right)$, the maximum $U_{\text {mod }}$ is $45 \mu \mathrm{V}$ at $4.2 \mathrm{~K}$.

${ }^{\text {a)} E l e c t r o n i c ~ m a i l: ~ m . p e d y a s h @ t n . u t w e n t e . n l ~}$
In Fig. 3 the experimental data for the temperature dependence of the voltage modulation $U_{\text {mod }}(T)$ are given. $U_{\text {mod }}(T)$ of the nanobridge SQUID is essentially different from the one of conventional weak link SQUIDs described by the RSJ model. For wider bridges with decreasing temperature starting from $T_{c}$ of the bulk material, the amplitude of the modulation increases rapidly, and reaches quite a narrow maximum at $T \approx 0.9 T_{c}$. With further decrease of temperature, the amplitude of modulation drops and vanishes, remaining zero at lower temperature. Narrower bridges, where $T_{c}$ is suppressed significantly, show a maximum at higher temperature, a decrease of the voltage modulation in intermediate temperature range, and an abrupt raise at low temperatures (typically below $20 \mathrm{~K}$ ) (see also Fig. 5). This behavior cannot be explained by an increase of the SQUID screening parameter $\beta_{L}=2 I_{c} L_{\mathrm{sq}} / \Phi_{0}$ due to the $I_{c}$ increase with decreasing temperature. The increase of $\beta_{L}$ leads to a saturation of the modulation amplitude and not to a suppression.

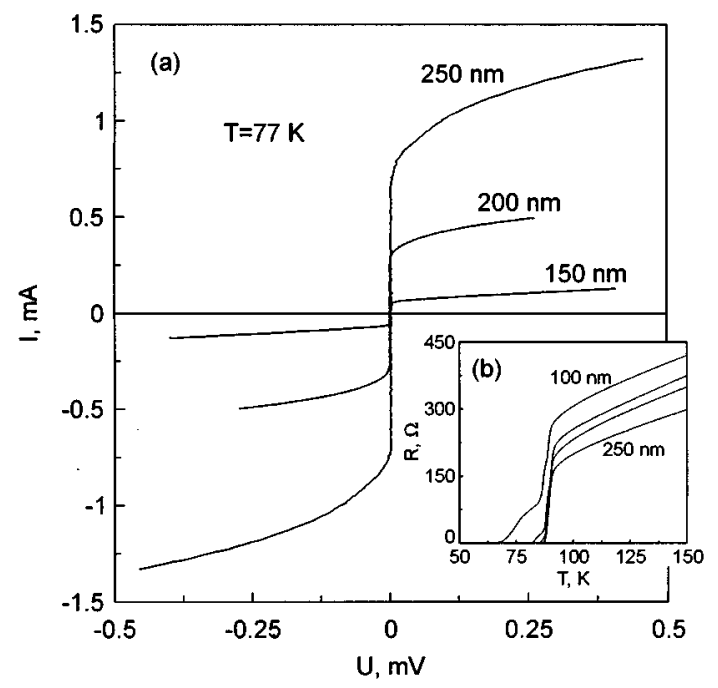

FIG. 1. (a) The $I(V)$ and (b) $R(T)$ characteristics of SQUIDs with nanobridges of different widths. 


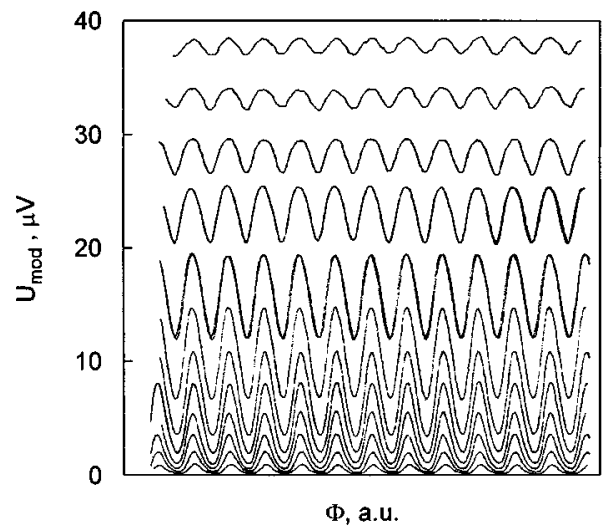

FIG. 2. A typical voltage-flux modulation of a nanobridge SQUID for different bias voltages (here, $w=250 \mathrm{~nm}$ and $T=77.4 \mathrm{~K}$ ).

It is reasonable to assume that superconducting properties of $\mathrm{YBaCuO}$ change in the vicinity of a trench patterned by either FIB or EBL. Earlier experiments demonstrated that a superconductor shows degradation of $T_{c}$ after irradiation by high energy ions. ${ }^{6}$ We assume the spatial distribution of $T_{c}$ in a region close to the trench follows the formula:

$$
T_{c}(x)=T_{\mathrm{cb}}\left(1-e^{-X / X_{0}}\right),
$$

where $T_{\mathrm{cb}}$ is the critical temperature of bulk, $x$ is the distance from the trench, and $x_{0}$ is the characteristic length of the $T_{c}$ variation. Applying this formula to nanobridges (i.e., two trenches at distance $w=2 x$ ), decrease of $T_{c}$ of the nanobridge with decreasing $w$ is expected. This is confirmed by the experimental results [see Fig. 1(b)], taking $x_{0}$ in the order of $50 \mathrm{~nm}$.

Let us assume that a nanobridge behaves like an SNStype junction in the temperature range $T_{c 2}<T<T_{c 3}$, and like a bulk superconductor below $T_{c 2}$, where $T_{c 2}$ is the critical temperature of the central region of the bridge, and $T_{c 3}$ is $T_{c}$ of the layer next to it (see Fig. 4). Such system can be described by the "two fluid" model, where the Josephson component of the supercurrent $I_{j}$ is gradually substituted by a "strong" term (i.e., nonperiodic with the phase difference $\Delta \varphi)$ with decreasing temperature. We calculated the tempera-

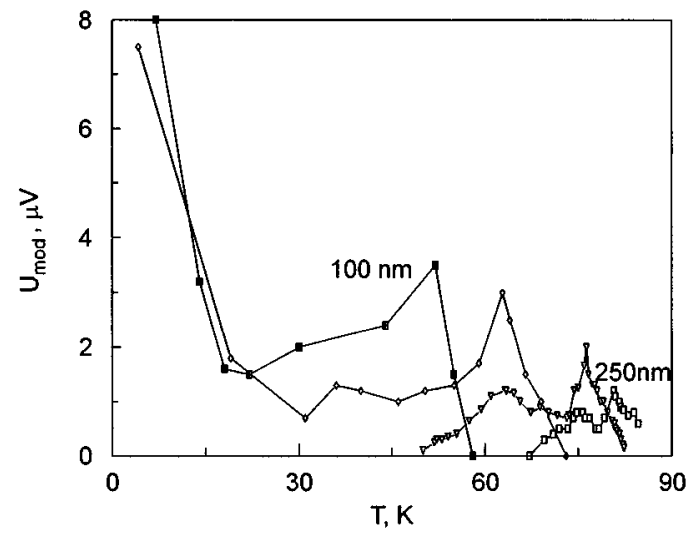

FIG. 3. Voltage-flux modulation $U_{\text {mod }}$ as a function of temperature for SQUIDs with bridges of different widths ( $w=100,150,200$, and $250 \mathrm{~nm}$ ).

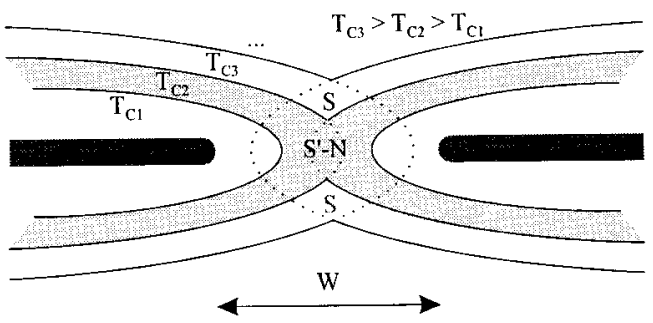

FIG. 4. Schematic critical temperature distribution inside the nanobridge area.

ture dependence $I_{j}$ [see Fig. 6(a)] using an expression derived by Likharev and Kupriyanov et al. ${ }^{7,8}$ for long $\mathrm{SS}^{\prime} \mathrm{S}$ junctions:

$$
I_{j}=\frac{\text { const } I_{c}(T)}{\left(1+\sqrt{1+A^{2} / 2}\right)^{2}} \exp \left(-\frac{L}{\xi}\right) .
$$

Here, $A^{2}=\left(T_{c s}-T\right) /\left|T_{c}-T\right|$, where $T_{c s}$ is the critical temperature of the junction electrodes and $T_{c}$ is that of the junction area itself. $L$ is the length of the junction. We substituted in the model $T_{c s}$ by $T_{c 3}$ and $T_{c}$ by $T_{c 2}$, where both $T_{c 2}$ and $T_{c 3}$ are functions of the bridge width $w$ according to Eq. (1).

The SQUID voltage modulation is proportional to the critical current $I_{c}$ (assumed to be the Josephson current) and can be written as ${ }^{9}$

$$
U_{\mathrm{mod}}=\frac{7}{\pi^{2}} \frac{I_{c} R_{n}}{1+\beta_{L}}\left(1-3.57 \frac{\sqrt{k_{b} T L_{\mathrm{sq}}}}{\Phi_{0}}\right) .
$$

This expression explains the vanishing of $U_{\bmod }$ of the SQUIDs with decreasing temperature due to the decline of the Josephson term $I_{j}$ of the full supercurrent of the nanobridge. By assuming a $T_{c}$ distribution along the bridge according to Eq. (1), the temperature range where the modulation is observed can be calculated and agrees with the experiment for bridges of all widths.

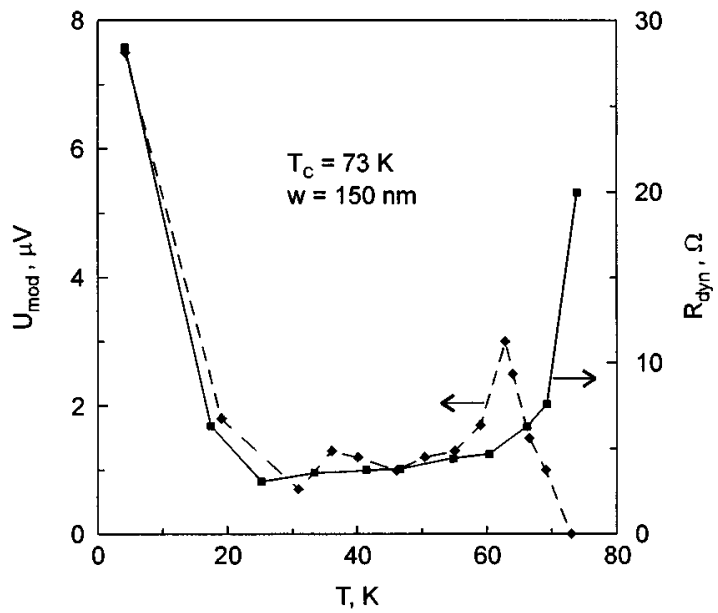

FIG. 5. The amplitude of the SQUID voltage-flux modulation $U_{\bmod }$ and its dynamic resistance $R_{\text {dyn }}\left(U_{\text {bias }}=10 \mu \mathrm{V}\right)$ as a function of temperature. 


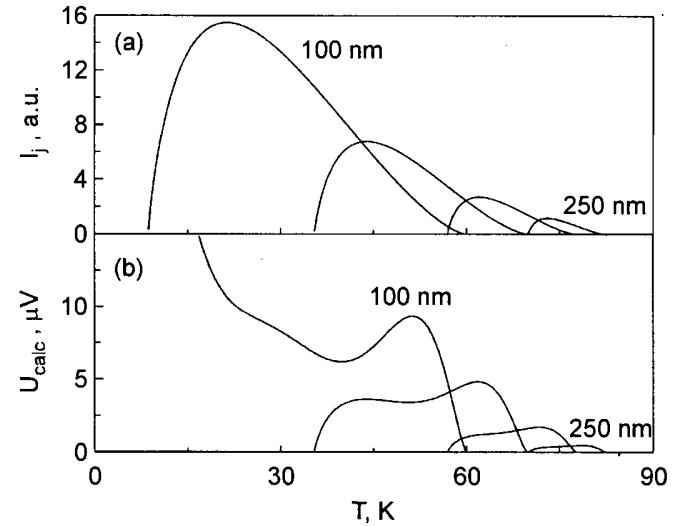

FIG. 6. Calculated Josephson current component $I_{j}$ (a) and the amplitude of the voltage-flux modulation $U_{\text {calc }}$ (b) for SQUID with bridges of different widths $(w=100,150,200$, and $250 \mathrm{~nm})$.

The difference between our SQUIDs and the conventional Josephson weak link devices described by the RSJ model is that the nanobridge SQUID is a typical "flux-flow" device with the corresponding $I-V$ characteristics [see Fig. 1(a)]. Instead of a single and well-defined RSJ-like resistance parameter $R_{n}$, the nanobridge SQUID is characterized by its bias-dependent dynamic resistance $R_{\mathrm{dyn}}$, which is determined by effects of vortex flow in the bridge and can depend on temperature in a rather complicated way. In Fig. 5, $R_{\text {dyn }}$ of a SQUID with $150 \mathrm{~nm}$ bridges, measured experimentally, is presented. It raises strongly in both low and high temperature regions. By substituting in Eq. (3) $R_{n}$ the approximation of the experimental values of $R_{\mathrm{dyn}}$ and $I_{c}$ by the calculated $I_{j}(T)$, the SQUID voltage modulation $U_{\text {calc }}$ was calculated as a function of temperature for bridges of different width [Fig. 6(b)]. A qualitative agreement between the experimental data and the calculated ones has been obtained for all temperatures and bridge widths (compare Figs. 3 and 6(b)].

A more realistic assumption would be that even at high temperatures, only a small fraction of total supercurrent in the bridge is of Josephson origin. This coincides with the high observed values of $J_{c}$ at $77 \mathrm{~K}$ and explains why we did not see any significant suppression of $I_{c}$ by a weak magnetic field. The above discussion brings up the idea that vortices in the nanobridge are not always of the pure Abrikosov type, but can gain properties of Josephson fluxons, like increase of the vortex size and gradual disappearance of the normal vortex core with decreasing $w .{ }^{7}$ This could also explain the low value of the viscous drag coefficient $\eta$ of vortex motion in a nanobridge..$^{5}$ The proposed $T_{c}(x)$ distribution near the bridge edge leads to a more complicated shape of the edge barrier for a vortex to enter the bridge than usually assumed. ${ }^{4}$

In summary, SQUIDs based on high- $T_{c}$ thin films have been made. They show well-reproducible voltage-flux modulation $U_{\text {mod }}$ with amplitude comparable to that of conventional weak link SQUIDs. The temperature dependence of $U_{\text {mod }}$ is found to be essentially different from that known for weak link SQUIDs. To explain this phenomenon, we considered degradation of superconductor in the nanobridge area, which leads to a local suppression of $T_{c}$ and to a transition from SNS to SS'S-type junctions with decreasing temperature. This approach allows us to explain the experimental data for $T_{c}$ and $U_{\text {mod }}(T)$ for SQUIDs with nanobridges of different widths.

${ }^{1}$ S. E. Romaine, P. M. Mankiewich, W. J. Skocpol, and E. Westerwick, Appl. Phys. Lett. 59, 2603 (1991).

${ }^{2}$ J. Schneider, M. Mück, and R. Wördenweber, Appl. Phys. Lett. 65, 2475 (1994).

${ }^{3}$ D. H. A. Blank, W. Booij, H. Hilgenkamp, B. Vulink, D. Veldhuis, and H. Rogalla, IEEE Trans. Appl. Supercond. 5, 2786 (1995).

${ }^{4}$ H. Rogalla, Habilitation thesis, University of Giessen, Germany (1986).

${ }^{5}$ M. V. Pedyash, G. J. Gerritsma, D. H. A. Blank, and H. Rogalla, IEEE Trans. Appl. Supercond. 5, 1387 (1995).

${ }^{6}$ S. S. Tinchev, IEEE Trans. Appl. Supercond. 3, 28 (1993).

${ }^{7}$ K. K. Likharev, Rev. Mod. Phys. 51, 101 (1979).

${ }^{8}$ M. Yu. Kupriyanov, K. K. Likharev, and V. F. Lukichev, Sov. Phys. JETP 56, 235 (1982).

${ }^{9}$ K. Enpuku, Y. Shimomura, and T. Kisu, J. Appl. Phys. 73, 7929 (1993). 